\title{
HIGHLIGHTS
}

ENDOSCOPY

\section{Acoustic lubrication reduces pain and discomfort associated with nasogastric intubation}

A novel nano vibrator system that uses surface acoustic waves has been shown to ease discomfort and pain associated with nasogastric intubation.

Many patients who undergo nasogastric intubation experience significant trauma,

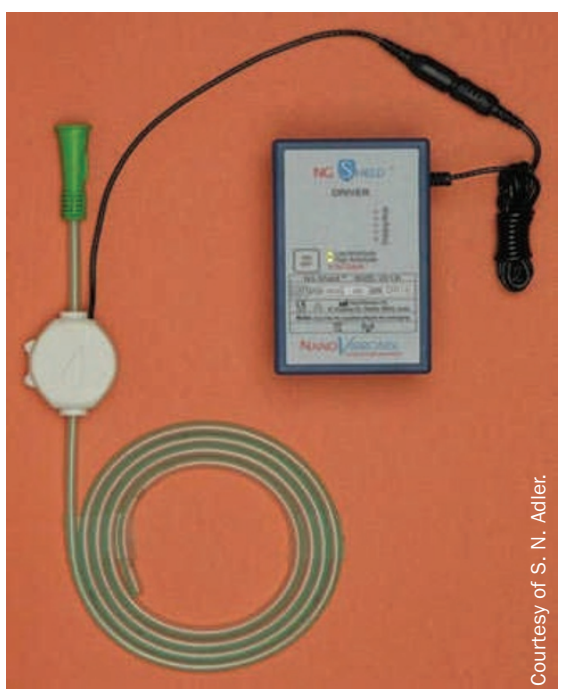

discomfort and pain related to both the insertion of the tube and the period of indwelling. Friction between the tube and nasal and pharyngeal mucosa is thought to be responsible for these adverse effects and the use of lubricating jelly has been advocated to relieve discomfort. However, as lubricating jelly is absorbed quickly it is unable to decrease friction between the tube and tissue mucosa when the tube is in place.

The NG-Shield device designed by Schlager and colleagues uses vibration of the nasogastric tube at a frequency of between $20-50 \mathrm{kHz}$ to reduce discomfort. This vibration causes the tube and mucosal tissue to be in contact for only half of the usual amount of time, thereby reducing friction. The acoustic waves are also intended to decrease the adhesion effect of the tube on the nasopharynx, which causes irritation when the tube is in situ.

The device was initially tested in vitro and a $26 \%$ reduction in the coefficient of friction was reported, leading Schlager and colleagues to hypothesize that this technology may indeed reduce pain.

A single-center, crossover, blinded study of 24 healthy volunteers confirmed that the hypothesis was correct. Each volunteer had a nasogastric tube inserted for $6 \mathrm{~h}$, during which time they were asked to rate their experience of pain and discomfort during periods of device activation and deactivation. The NG-Shield was shown to significantly reduce pain and discomfort in the nose and throat. The authors now plan to confirm these findings in patients undergoing nasogastric intubation in clinical practice.

\section{Rachel Thompson}

\footnotetext{
Original article Schlager, A. et al. Use of surface acoustic waves to reduce pain and discomfort related to indwelling nasogastric tube. Endoscopy doi:10.1055/s-0030-1255801.
} 